\title{
ADAPTIVE ROUTING SCHEME FOR RELIABLE COMMUNICATION IN VEHICULAR AD-HOC NETWORK (VANET)
}

\author{
Karthikeyini S. SIVASUBRAMANIAN ${ }^{1^{*}}$, Shankar S. SUBRAMANIAM ${ }^{2}$ \\ ${ }^{1}$ Faculty of Faculty of Information and Communication Engineering, Anna University, India \\ ${ }^{2}$ Dept of Computer Science and Engineering, Hindusthan College of Engineering and Technology, India
}

Received 2 January 2019; revised 4 April 2019, 3 June 2019; accepted date 29 June 2019; first published online 5 June 2020

\begin{abstract}
In a wireless communication system, due to the presence of the surrounding objects, the amplitude of the received signal rapidly changes by reflection, diffraction, and scattering and noise is added to the received signal. This prompts multipath fading and interference, which affects the quality of communication. The proposed Adaptive Routing Scheme (ARS) considers the algorithm Reliable Routing (RR) using Average Bit Error Rate expressed in Nakagami-m fading channel (ABERN-m) to predict the quality of the link, the Energy Efficient Routing (EER) calculates Remaining Battery Energy (RBE) to extend the network lifetime. The Canberra Distance Measure (CDM) is used instead of Euclidean Distance Measure (EDM) to improve the accuracy of distance measurement in mobile nodes. The aim of the proposed scheme is to predict the best optimal path and maintain the consistent path to enhance the Quality of Service (QoS) in real-time communication to improve efficient traffic on the road.
\end{abstract}

Keywords: average bit error rate expressed in Nakagami-m fading channel (ABERN-m), adaptive routing scheme (ARS), energy efficient routing (EER), Canberra distance measure (CDM), Euclidean distance measure (EDM).

\section{Notations}

ABER - average bit error rate;

ABERN- $m$ - average bit error rate expressed in Nakagami-m fading channel;

ARS - adaptive routing scheme;

AU - application unit;

AWGN - additive white Gaussian noise;

BPSK - binary phase shift key;

$\mathrm{CCH}$ - control channel;

$\mathrm{CDM}$ - Canberra distance measure;

$\mathrm{CH}$ - cluster head;

CLAO-TCP - cross layer aware optimization of transfer control protocol;

CSMA/CA - carrier sense multiple access/collision avoidance;

DSRC - dedicated short-range communication;

EDM - Euclidean distance measure;

EED - end-to-end delay;

EER - energy efficient routing;

EU - energy usage;

FCC - federal communication commission;
G-TDMA - generalized time division multiple access;

IETF - internet engineering task force;

IPv6 - internet protocol version 6;

ITS - intelligent transportation system;

LLC - logical link control;

LOS - line of sight;

MAC - medium access control;

MGF - moment generating function;

NLOS - no-line-of-sight;

OBU - on board unit;

PDF - probability density function;

PDR - packet delivery ratio;

PLR - packet loss rate;

QoS - quality of service;

R2R - RSU to RSU;

$\mathrm{RBE}$ - remaining battery energy;

$\mathrm{RO}$ - routing overhead;

$\mathrm{RR}$ - reliable routing;

RREP - route reply;

${ }^{*}$ Corresponding author. E-mail: karthikeyinicse@gmail.com 


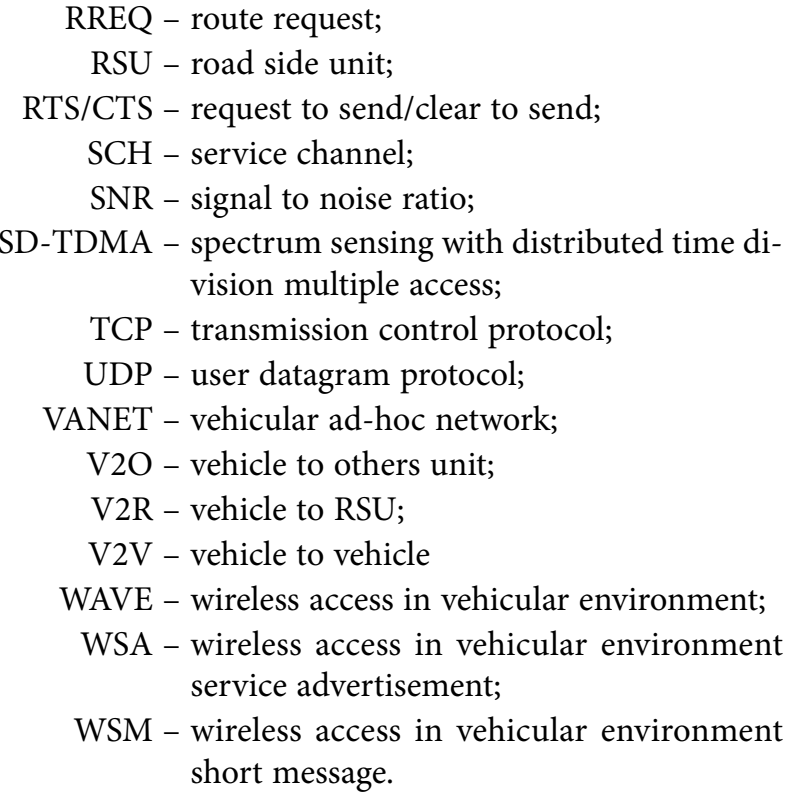

\section{Introduction}

VANET is a crucial part of ITS. It provides valuable information to assist the driver in order to prevent mishaps and congestion on the road (Sussman, Bronzini 2006). The VANET has a safety application feature, which provides, emergency brake light warning, collision warning, road curve warning and so on. It also has non-safety application, like, navigation, traffic information, and internet access. The DSRC regulation dictated by the US FCC and is designed for $75 \mathrm{MHz}$ transmission capacity and $5.9 \mathrm{GHz}$ data transmission for intelligent transportation application services. The FCC authorized the operation of DSRC spectrum from 5.850 to $5.925 \mathrm{GHz}$ (IEEE Standard $802.11 \mathrm{p}-2010)$. This spectrum consists of $5 \mathrm{MHz}$ received as a guard band at the lower end of DSRC spectrum and the remaining $70 \mathrm{MHz}$ is divided into six $\mathrm{SCH}$ and one $\mathrm{CCH}$. The channel 178 intended for $\mathrm{CCH}$ is utilized for the WSM and WSA, and SCH is utilized for IPv6 packets and WSM. The optional assignment of the $20 \mathrm{MHz}$ for channel 175 and 181 is allocated to critical safety message. These channels are located on either side of Channel 178, which is illustrated in Figures 1 and 2.

\begin{tabular}{|l|rrr|}
\hline \multicolumn{4}{|c|}{ Frequency [GHz] } \\
5.470 & 5.82 & 5.850 & 5.925 \\
\hline \multicolumn{2}{|c|}{ Unlicensed WiFi/WiMax } & & DSRC \\
\hline
\end{tabular}

Figure 1. Unlicensed and licensed DSRC band

\begin{tabular}{|c|c|c|c|c|c|c|c|}
\hline \multicolumn{2}{|c|}{$\overbrace{}^{\text {Reserved }}$} & \multicolumn{2}{|c|}{ Ch $175(20 \mathrm{MHz})$} & & \multicolumn{2}{|c|}{ Ch 181 (20 MHz) } & \\
\hline & $\begin{array}{c}\mathrm{CH} 172 \\
\text { SCH }\end{array}$ & $\begin{array}{l}\mathrm{CH} 174 \\
\mathrm{SCH}\end{array}$ & $\begin{array}{l}\mathrm{CH} 176 \\
\text { SCH }\end{array}$ & $\begin{array}{c}\mathrm{CH} 178 \\
\text { SCH }\end{array}$ & $\begin{array}{l}\mathrm{CH} 180 \\
\text { SCH }\end{array}$ & $\begin{array}{l}\text { CH } 182 \\
\text { SCH }\end{array}$ & $\begin{array}{c}\mathrm{CH} 184 \\
\mathrm{SCH}\end{array}$ \\
\hline J & & 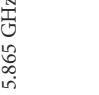 & & & 6 & & \\
\hline
\end{tabular}

Figure 2. US DSRC band plan

\section{DSCR for VANET}

The IEEE Standard 802.11-2016 has been amended to support DSRC communication. Approved amendments to the IEEE Standard 802.11-2016 referred to as IEEE Standard $802.11 \mathrm{p}-2010$ is used to support WAVEs. The MAC sublayer of the link layer is defined by IEEE $802.11 \mathrm{p}$ (Sivasakthi, Suresh 2013). The MAC extension protocol is defined by the IEEE Standard 1609.4-2016, which supports a device to switch among the various DSRC channels allocated by the FCC. The LLC sublayer is defined by the IEEE Standard 802.2-1989. In safety applications, IEEE Standard 1609.3-2016 defines WSM, WSA and protocols for the network and transport. The IEEE Standard 1609.2b-2019 is used for security to encrypt and authenticate the messages. In non-safety applications, IETF defines the TCP or UDP for transport layer services and it defines an IPv6 for network layer services. The non-safety applications run over the internet and DSRC. The safety application developed by the SAE Standard J2735_201603 application is exemplified in Figure 3.

\subsection{Component of VANET}

DSRC system equipment is an OBU, which has a highly mobile node and a RSU, which is fixed near the roadside. Each vehicle is composed of $\mathrm{OBU}$ and an $\mathrm{AU}$, which is connected through a wired or wireless medium (Al-Sultan et al. 2014). There are four types of communication: $\mathrm{V} 2 \mathrm{~V}$, V2R, R2R via IEEE Standard $802.11 \mathrm{p}-2010$ and V2O via IEEE Standard 802.11a-1999, IEEE Standard 802.11b1999 and IEEE Standard IEEE 802.11g-2003. An OBU can communicate with the Internet through RSU or HS and RSU can communicate with the Internet through the gateway (Hartenstein, Laberteaux 2008), which is illustrated in Figure 4.

\subsection{Current issues and challenges in the communication}

Road accidents have become a serious issue due to the increase in the number of vehicles in the recent years, which

\begin{tabular}{|c|c|}
\hline $\begin{array}{l}\text { Safety application } \\
\text { SAE J2735 }\end{array}$ & Non-safety application \\
\hline $\begin{array}{c}\text { Transport and network } \\
\text { layer - WSPM }\end{array}$ & $\begin{array}{c}\text { Transport layer - TCP/UDP } \\
\text { IETF RFC 793/768 }\end{array}$ \\
\hline $\begin{array}{l}\text { IEEE } 1609.3 \\
\text { security } \\
\text { IEEE } 1609.2\end{array}$ & $\begin{array}{l}\text { Network layer - IPv6 } \\
\text { IETF RFC } 2460\end{array}$ \\
\hline \multicolumn{2}{|c|}{$\begin{array}{l}\text { LLC sublayer } \\
\text { IEEE } 802.2\end{array}$} \\
\hline \multicolumn{2}{|c|}{$\begin{array}{l}\text { MAC sublayer extension } \\
\text { IEEE } 1609.4\end{array}$} \\
\hline & er $\}$ IEEE $802.11 \mathrm{p}$ \\
\hline
\end{tabular}

Figure 3. DSRC standard in the US 


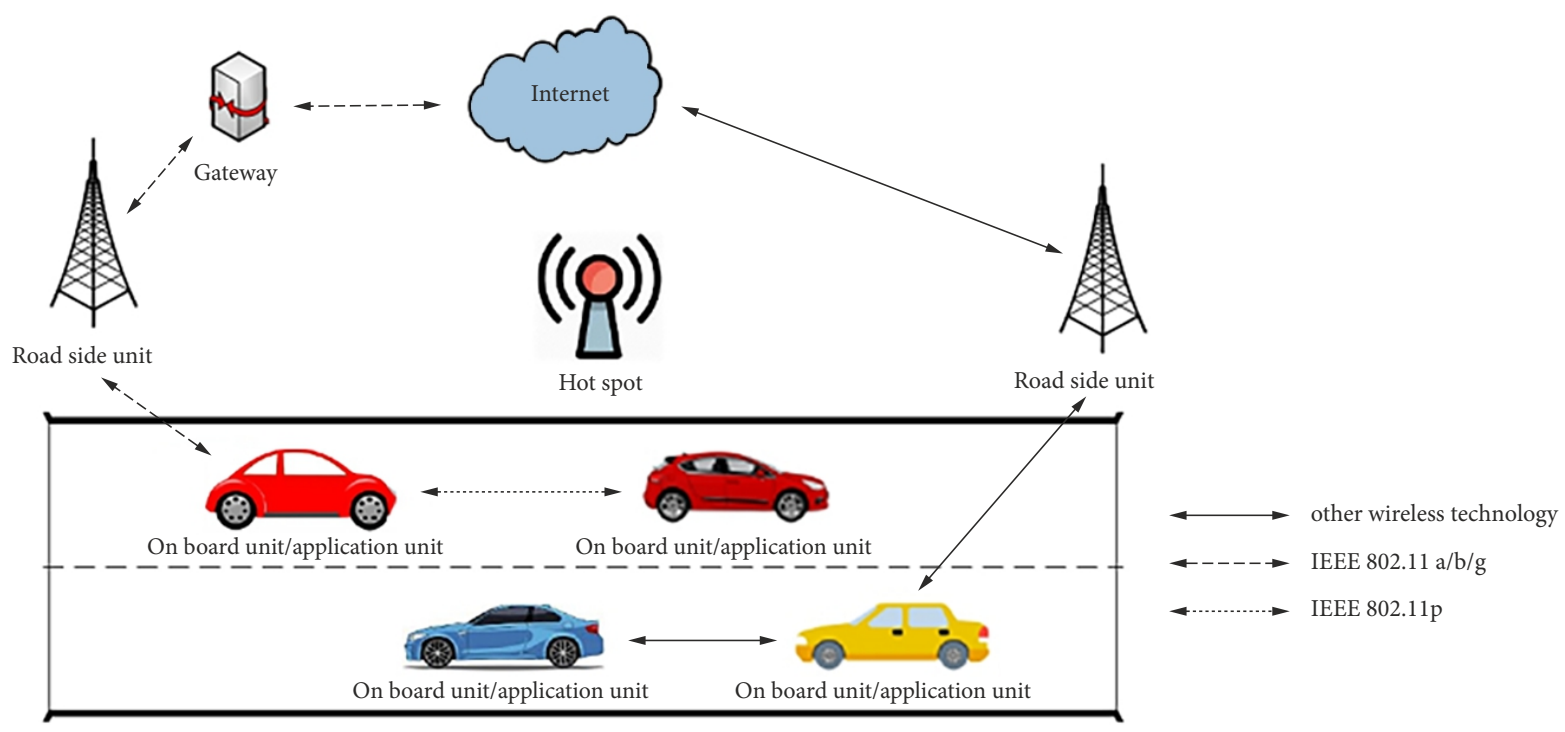

Figure 4. Architecture of VANET

results in loss of human life and property. The emerging technology of vehicular communication system can be more effective in avoiding accidents and traffic congestion. To improve the safety of the passengers travelling on the road and to enhance the traffic efficiency, the ITS provides an application which gives a warning signal such as the condition of the roads, road intersection, collision avoidance, traffic management, toll services, and comfort applications. In VANET, RSUs and vehicles able to communicate through different wireless communications depending on the different applications.

There are some challenging tasks in VANET. First, the network topology is frequently changed and connectivity of nodes is frequently disconnected due to the high mobility nature of VANET. This makes it hard to predict the node position. The dynamic topology nature of the VANET should maintain dynamic routing to improve the quality of communication. Secondly, the realistic communication channel needed to measure the actual received signal strength. Since the vehicles are travelling through different traffic scenario and unpredictable environment, we have to design the realistic communication channel model to cover various types of traffic and environmental conditions. As the ITS safety application must be delivered to the neighbouring vehicles within a time limit, we should well maintain the quality of communication between the vehicles.

\section{Related works}

Since the transmitted signal in the wireless communication medium is attenuated by reflection, diffraction, and scattering we need a radio propagation model to predict the actual signal strength at the receiver to predict the link quality. In physical layer, to measure the fading effect, the existing large-scale fading model is used to measure the received power. The received power is varying gradually due to signal attenuation cost by the deterministic metrics of distance, obstacle, and environmental property (Martinez et al. 2009). This model ignores the rapid fluctuation of instantaneous received power in small area due to multipath effects. The proposed work considers the challenging task of the randomness of the mobility of the vehicle, and unpredictability of the surrounding environment. We need a small-scale propagation model to measure the signal strength at the receiver within small distance or a lesser time interval.

In the routing process, there are some links which are more reliable than the others and hence they are frequently used to forward many packets. The nodes along the reliable link will be overused without considering the RBE. Since the overused node will consume more energy there are chances of rapid failure of the node, which will degrade the performance of the entire network (Gomez et al. 2003). Since all nodes in the network are operated using batteries and the lifetime of nodes depend upon its batteries, the RBE is a critical requirement in wireless adhoc network. The sector based energy efficient adaptive routing algorithm (Dhanapal, Visalakshi 2015a, 2015b) finds the RBE of node to avoid the overuse of the node and improve the network lifetime. But, they do not address the transmitted signal attenuated by different fading parameters. In real time applications, all nodes should be continuously alive and reliable to improve the performance of the entire network. The proposed work intends to accomplish higher reliability and maintain the RBE in the routing to improve the QoS over the network.

\section{Proposed system}

The proposed system, ARS addresses three categories. The first category algorithm RR includes the reliability of the node by finding ABER of receiving signal from the transmitted signal by using Nakagami-m fading channel. The existing large-scale communication channel model (Hrovat et al. 2014) is used to measure the received sig- 
nal strength at a given distance and surrounding environment, which is expressed in the Equation (1):

$$
y=x \cdot h+n,
$$

where: $y$ is the received signal strength; $x$ is the transmitted signal; $h$ is the path-loss fading effect that attenuates the transmitted signal gradually in wireless medium; $n$ is the fixed noise added to the transmitted signal.

This propagation model usually predicts the average received signal from the transmitter at a given distance and fixed surrounding environment. Due to the unpredictable mobility of the vehicle and surrounding environment, the small-scale communication model is used. The proposed small-scale communication channel model (Rappaport 2002) used to measure the rapid fluctuation of the signal from the transmitter, which is expressed in the Equation (2):

$$
y=x \cdot h_{1}+n,
$$

where: $y$ is the received signal of the node; $x$ is the transmitted signal; $h_{1}$ is the multipath fading effect that attenuates the transmitted signal rapidly in the wireless medium; $n$ is the fixed noise added to the transmitted signal.

This propagation model measures the average received signal in the unpredictable mobility of the vehicle and surrounding environment. The first category algorithm RR calculates the ABERN-m to measure the multipath fading effect is to key out the reliability of path over the network. The second category algorithm EER calculates the energy efficiency of route by finding the RBE of nodes in routing to order to avoid the overuse of node and improve the network lifetime. The third category algorithm CDM is used instead of EDM to measure the accurate distance between the vehicles. The proposed system addresses the requirement of reliable, EER and accurate distance measure to maintain the consistent path in the entire network.

\section{Information model}

\subsection{RR scheme}

In a wireless ad-hoc communication, multipath fading occurs due to delayed, reflected, scattered, and diffracted signal components. Multipath fading is relatively fast and is responsible for the short-term signal variations. The transmitted signal is propagated in multipath fading by surrounding objects (Yacoub et al. 2005). The transmitted signal is attenuated by multipath fading effect and noise also added into the received signal. The wireless fading channel coefficient expressed in Equation (3):

$$
\begin{aligned}
& y=x \cdot h_{1}+n ; \\
& h_{1}=r \cdot \exp (\theta),
\end{aligned}
$$

where: $y$ is the received signal in wireless medium; $x$ is a transmitted signal; $r$ is the fading channel coefficient; $\theta$ is an angle which is uniformly distributed on $[-\pi, \pi] ; n$ is an AWGN, which is fixed SNR. The received signal strength is attenuated by the fast fading effect such as reflection, refraction and scattering due to surrounding objects.
In Nakagami-m fading channel, different values of $m$ are used to measure the different fading environments in a small geographical area (Beaulieu, Cheng 2005). PDF of the received signal power for Nagakami-m fading environment in a static wireless system (Govindan et al. 2011) is expressed in the Equation (4):

$$
\begin{aligned}
& f_{r}(r)=\frac{m^{m} \cdot r^{m-1}}{\Gamma(m) \cdot \Omega^{m}} \cdot \exp \left(-\frac{m \cdot r}{\Omega}\right) ; \\
& \frac{1}{2} \leq m<\infty ; 0 \leq r<\infty,
\end{aligned}
$$

where: $m$ is the fading parameter; $r$ is the random variable of instantaneous received signal strength; $\Omega$ is the average signal power; $\Gamma(m)$ is an Euler Gamma function.

The parameters $\Gamma(m), \Omega$ and $m$ are expressed in Equations (5)-(7).

If $m$ is integer:

$$
\begin{aligned}
& \Gamma(m)=(m-1) ! ; \\
& \Omega=E\left\lceil r^{2}\right\rceil,
\end{aligned}
$$

where: $r=r_{1}+r_{2}+\ldots+r_{N}$ that are sample of the received envelop to estimate the fading parameter $m ; E\left\lceil r^{2}\right\rceil$ is a average fading power.

The fading parameter $m$ defines the severity of fading in the communication channel (Tepedelenlioglu, Gao 2005):

$$
m=\frac{\Omega^{2}}{E \cdot\left(r^{2}-\Omega\right)^{2}} .
$$

To improve the simplicity of the Equation (4), it can be written in MGF (Simon, Alouini 2002, 2008), which is expressed in Equation (8):

$$
M_{r}(-s)=\left(1-\frac{s \cdot \Omega}{m}\right)^{-m}, s>0 .
$$

Due to the unpredictability of mobility and the surrounding environment, the proposed work considers probabilistic model that is Nakagami-m propagation model. This method is used to measure the received signal strength in multipath condition in order to measure the performance of the network. The Nagakami-m fading channel, shape parameter $m$ is easily configurable and covers different fading environmental conditions in the mobile ad-hoc wireless medium (Tarique, Hasan 2011). When the value of $m=1$, it becomes a Rayleigh distribution, which has NLOS between sender and receiver. When $m=1 / 2$ it becomes a one-sided Gaussian distribution. The fading effect of signal amplitude is more severe than a Rayleigh distribution when $m<1$. When $m>1$, the fading effect is less severe than Rayleigh fading. Nakagami-m distribution closely approximates the Rician distribution when $m=1$. The Nagakami and Rician distributions can be mapped to each other by the fading shape parameter $m$ and Rician $k$ factor. The value of $m$ applied to the Nakagami-m distribution, which is used to measure. The below Equation (9) indicates the different fading environments 
such as one-sided Gaussian, Rayleigh, and Rician distributions (Tiwari et al. 2009), when assigning different values of $m$ in Nakagami-m distribution:

$m= \begin{cases}\frac{1}{2}, & \text { one-sided Gaussian distribution; } \\ 1, & \text { Raylegh distribution, NLOS; } \\ >1, & \text { Rician distribution, } m=\frac{(k+1)^{2}}{(2 \cdot k+1)}, k=\frac{\sqrt{m^{2}-m}}{m-\sqrt{m^{2}-m}}, \text { LOS; } \\ \infty, & \text { no fadding occured. }\end{cases}$

The performance of the receiver in the dynamic wireless system is measured by the parameter called ABER, which is defined as the ratio of the number of wrong bits per second to the number of total bits transmitted per second. Since the distance between transmitter and sender is a random variable, ABER in ABERN-m is an important performance metric in measuring the quality of links (Craig 1991) which is expressed in Equation (10):

$$
A V G_{B E R}=\int_{0}^{\infty} p_{e}(r) \cdot f_{r}(r) \mathrm{d} r
$$

where: $A V G_{B E R}$ is average bit error rate; $p_{e}(r)$ is a fixed SNR (a probability of error symbol represented in AWGN); $f_{r}(r)$ is an instantaneous SNR.

It is represented in a Gaussian $Q$-function (Lever 1998). The probability of error of AWGN channel is expressed in Equation (11):

$$
p_{e}(r)=Q(a)=\frac{1}{\pi} \cdot \int_{0}^{\frac{\pi}{2}} \exp \left(-\frac{a^{2}}{2 \cdot \sin ^{2} \theta}\right) \mathrm{d} \theta, a \geq 0 .
$$

Information of bits is modulated prior to the transmission over the channel. Where $a$ is a constant, which depend on the specific modulation combination. One of the digital modulation formats BPSK used. The probability of bit error for BPSK modulation (Goldsmith 2005) in AWGN channel is expressed in Equation (12):

$$
\begin{aligned}
& p_{e}(r)=Q(a)=Q(\sqrt{2 \cdot r})= \\
& \frac{1}{\pi} \cdot \int_{0}^{\frac{\pi}{2}} \exp \left(-\frac{r}{\sin ^{2} \theta}\right) \mathrm{d} \theta, x \geq 0 .
\end{aligned}
$$

By substituting the Equation (12) in the Equation (10), we arrive at Equations (13) and (14):

$$
\begin{aligned}
& A V G_{B E R}=\int_{0}^{\infty} \frac{1}{\pi} \cdot \int_{0}^{\frac{\pi}{2}} \exp \left(-\frac{r}{\sin ^{2} \theta}\right) \mathrm{d} \theta f_{r}(r) \mathrm{d} r \\
& A V G_{B E R}=\frac{1}{\pi} \cdot \int_{0}^{\frac{\pi}{2}}\left(\int_{0}^{\infty} \exp \left(-\frac{r}{\sin ^{2} \theta}\right) f_{r}(r) \mathrm{d} r\right) \mathrm{d} \theta .
\end{aligned}
$$

The inner integral in the form of Laplace transform in the Equation (14) can be written as MGF, which is expressed in Equation (15):

$$
A V G_{B E R}=\frac{1}{\pi} \cdot \int_{0}^{\frac{\pi}{2}} M_{r}\left(-\frac{1}{\sin ^{2} \theta}\right) \mathrm{d} \theta
$$

where: $M_{r}\left(-\frac{1}{\sin ^{2} \theta}\right)$ is MGF of instantaneous fading
chanel.

By substituting the Equation (8) in Equation (15), the result is in Equation (16):

$$
A V G_{B E R}=\frac{1}{\pi} \cdot \int_{0}^{\frac{\pi}{2}}\left(1+\frac{\Omega}{m \cdot \sin ^{2} \theta}\right)^{-m} \mathrm{~d} \theta,
$$

when: $m=1$ the ABER in Rayleigh fading channel (Simon, Alouini 2002, 2008) is expressed in Equation (17):

$$
A V G_{B E R}=\frac{1}{2} \cdot\left(1-\sqrt{\frac{\Omega}{m+\Omega}}\right) .
$$

\subsection{Enhancement of network life time}

The main objective of routing to discover EER, which will prolong the lifetime of the network based on the weight of the RBE (Toh 2001) along with the reliable link. The RBE of node $n_{i}$ at time $t$ is expressed in Equation (18):

$$
R B E_{n_{i} t}=\text { initialenergy - consumed energy. }
$$

Weight of RBE is inversely proportional to the remaining energy of the batteries. The weight of the remaining energy of node calculated at different times, which is expressed in the Equation (19):

$$
W_{i}\left(R B E_{n_{i} t}\right)=\frac{1}{R B E_{n_{i} t}} .
$$

Let $\alpha$ be the threshold value of the ABER of a node and $\beta$ be a threshold value of the weight of remaining battery power of a node. We consider the set of nodes that have the threshold value of the ABER and the threshold power of batteries to find the optimal path. The sum of the life span of intermediate nodes on the optimal path is expressed in Equation (20):

$$
W_{i}(\text { optimal path } j)=\sum_{j=0}^{n} W_{i}\left(R B E_{n_{i} t}\right) \text {. }
$$

The maximum life span of the path is considered as a minimum value of the total weight of the all the paths and is expressed in Equation (21):

$$
\text { Best }_{\text {optimal path } j}=\min \left(W_{i}(\text { optimal path } j)\right) \text {. }
$$

where: 'min' is a function, which selects the minimum total weight of path to prolong the lifetime of the path.

The graph $\mathrm{G}$ contains set of path $j$ and set of node $n_{i}$ in path $j$. There are three paths $p_{1}, p_{2}, p_{3}$ from source to destination. As per Equation (19) total weight of optimal path $w\left(p_{1}\right)$ is $70, w\left(p_{2}\right)$ is 40 and $w\left(p_{3}\right)$ is 40 . According to the Equation (21) the best optimal path $p_{2}$ is selected for forward the packet from source to destination. If all the paths have the same total weight of the battery, the best optimal path is selected based on hop count, which is illustrated in the Figure 5. 


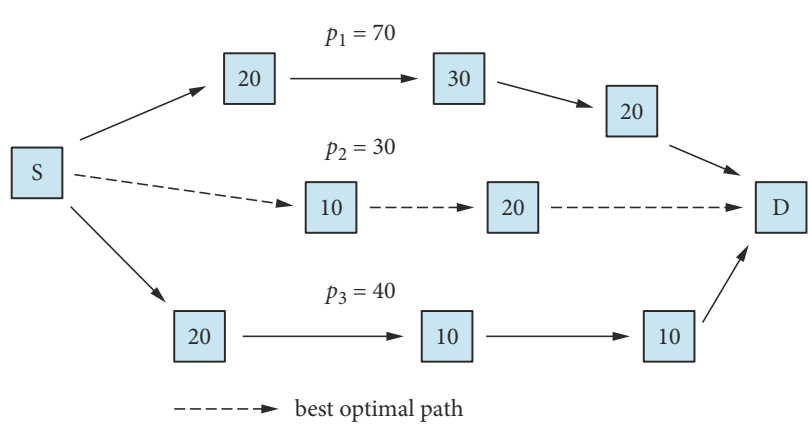

Figure 5. Best optimal path selection

\subsection{Accuracy improvement by $\mathrm{CDM}$}

The ITS is a novel technologies in the field of communication. To avoid the congestion on the road and to improve the traffic efficiency, it provides through the safety application such as route information, dangerous road conditions, safety overtake warning, accident sites information and emergency vehicle warning. Assume that an ambulance has to carry an injured person from the accident site to the hospital for emergency treatment. In such a scenario, the ambulance is allowed to break road rules like crossing the road when the traffic light is red or exceeding speed limit in order to reach the destinations in the right time. The ambulance broadcasts this emergency message to its neighbouring vehicle on the road to avoid mishaps and enable it to reach the destination at the earliest. The driver of the neighbouring vehicle will take timely action by receiving the emergency information. Each vehicle needs to find the neighbouring vehicle to disseminate the message. The distance measure metric must satisfy the following four conditions (Xu, Wunsch 2005):

1) distance between two coordinate must be non-negative, i.e. distance $\left(x_{i}, x_{j}\right)>0$ for all $x_{i}, x_{j}$;

2) if distance $\left(x_{i}, x_{j}\right)=0$, the two points are identical, i.e. $x_{i}=x_{j}$;

3) $\operatorname{distance}\left(x_{i}, x_{j}\right)=\operatorname{distance}\left(x_{j}, x_{i}\right)$;

4) distance measure must satisfy the triangular inequality, i.e. $\operatorname{distance}\left(x_{i}, x_{k}\right) \leq \operatorname{distance}\left(x_{i}, x_{j}\right)+$ $\operatorname{distance}\left(x_{j}, x_{k}\right)$.

The Euclidean distance satisfies the above metric condition so this EDM is metric. The coordinates of pair of object is calculated by using the formula of Euclidean distance is expressed in Equation (22). The example of coordinates of vehicles defined in Figure 6.

$$
\text { Distance }_{\text {Euclidean }}=\sqrt{\sum_{k=1}^{n}\left(x_{j k}-y_{j k}\right)^{2}} \text {. }
$$

The Bray-Curtis distance or Sørensen distance is semi metric, which is not satisfying the triangle inequality property. The drawback of this measure is undefined if both coordinates near to zero value (Bray, Curtis 1957). The proposed Canberra distance which used to measure the distance in mobile node (Lance, Williams 1966) is an extended version of Manhattan or City block distance.

\begin{tabular}{|c|c|c|c|}
\hline Vehicles & \multicolumn{3}{|c|}{ Coordinates of vehicles } \\
\hline V1 & 1 & 1 & 1 \\
\hline V2 & 1 & 1 & 0 \\
\hline V3 & 2 & 2 & 2 \\
\hline V4 & 10 & 10 & 10 \\
\hline V5 & 11 & 11 & 11 \\
\hline V6 & 10 & 5 & 0 \\
\hline
\end{tabular}

Figure 6. Coordinates of vehicles in the area

The CDM method examines the sum of series of fractional difference between the coordination of pairs of objects. The CDM is defined if both coordinate near zero value (Jain, Dubes 1988). The CDM is defined in Equation (23):

$$
\text { Distance }_{\text {Canberra }(i, j)}=\frac{1}{n} \cdot \sum_{k=1}^{n}\left|\frac{x_{j k}-y_{j k}}{x_{j k}+y_{j k}}\right| \text {. }
$$

The time complexity of both Euclidean distance and $\mathrm{CDM}$ are $O(n)$ in linear time, but the Canberra distance has more accuracy in measuring the distance of the mobile objects than Euclidean distance (Shirkhorshidi et al. 2015), which is defined in Table 1.

The Euclidean measure indicates the distance between the vehicles V1 and V3 is same as between V4 and V5. Since the CDM indicates the distance between the vehicles V1 and V3 is not same as between V4 and V5, this analysis indicates that the CDM is more sensitive for values closer to the origin.

\subsection{Proposed ARS RREQ format}

In the route selection process, among the available routes, the source node selects the best optimal path based on the weighted estimation of ABER and remaining battery capacity. The RREQ format of the proposed ARS protocol is shown in the Figure 7.

In type field, the bits 012 are used for precedence; bits 345 are used for type of service. Flag G is indicating whether RREP should be unicast to the destination IP address. Flag D indicates whether destination responding to the RREQ. Identification is a number to identify all fragments of the original IP packet. The hop count is the number of hops by which the message is carried from the source address to the destination address.

\section{ARS}

Finding the best optimal path from source to destination based on the ABERN-m fading channels, the remaining energy of batteries, and by calculating the accurate distance:

1) where $d$ is a distance, $d=\left(d_{0}, d_{1}\right), d_{0}=80 \mathrm{~m}$, $d_{1}=200 \mathrm{~m}$, ABER threshold $0 \leq \alpha \leq 0.3$ (Das et al. 2016 ), RBE threshold value $\beta$ is $12 \mathrm{~V}$; the distance between the pair of coordinate is calculated by CDM; 
Table 1. Distance between the vehicles measured by Euclidean and Canberra metrics

\begin{tabular}{|l|c|c|c|c|c|c|c|}
\hline EDM & V1 & V2 & V3 & V4 & V5 & Distance between V1 and V3 & Distance between V4 and V5 \\
\hline V1 & 0 & 1 & 1.732 & 15.588 & 17.321 & 1.732 & 1.733 \\
\hline V2 & 1 & 0 & 2.449 & 16.186 & 17.916 & 1.499 & 1.73 \\
\hline V3 & 1.732 & 2.449 & 0 & 13.856 & 15.588 & 1.732 & 1.732 \\
\hline V4 & 15.588 & 16.186 & 13.856 & 0 & 1.732 & 1.732 & 1.732 \\
\hline V5 & 17.321 & 17.916 & 15.588 & 1.732 & 0 & 1.733 & 1.732 \\
\hline V6 & 9.899 & 9.849 & 8.775 & 11.180 & 12.570 & 1.125 & 1.39 \\
\hline CDM & V1 & V2 & V3 & V4 & V5 & Distance between V1 and V3 & Distance between V4 and V5 \\
\hline V1 & 0 & 1.000 & 1.000 & 2.455 & 2.500 & & 0.045 \\
\hline V2 & 1.000 & 0 & 1.667 & 2.636 & 2.667 & 0.667 & 0.031 \\
\hline V3 & 1.000 & 1.667 & 0 & 2.000 & 2.077 & 1.000 & 0.077 \\
\hline V4 & 2.455 & 2.636 & 2.000 & 0 & 0.143 & 0.455 & 0.143 \\
\hline V5 & 2.500 & 2.667 & 2.077 & 0.143 & 0 & 0.423 & 0.143 \\
\hline V6 & 2.485 & 1.485 & 2.095 & 1.333 & 1.423 & 0.39 & 0.09 \\
\hline
\end{tabular}

$012345 \quad 6 \quad 7 \quad 01234567001234567 \quad 01234567$

\begin{tabular}{|c|c|c|c|c|c|}
\hline Type & G & $\mathrm{D}$ & \multicolumn{2}{|c|}{ Identification } & Hop count \\
\hline \multicolumn{3}{|c|}{ RREQ ID } & ABER & \multicolumn{2}{|c|}{ Remaining battery capacity } \\
\hline \multicolumn{6}{|c|}{ Destination address } \\
\hline \multicolumn{6}{|c|}{ Destination sequence number } \\
\hline \multicolumn{6}{|c|}{ Source address } \\
\hline \multicolumn{6}{|c|}{ Source sequence number } \\
\hline
\end{tabular}

Figure 7. RREQ format of ARS protocol

2) Distance ${ }_{\text {Canberra }}\left(x_{i}, x_{j}\right)=\frac{1}{n} \cdot \sum_{k=1}^{n}\left|\frac{x_{i k}-x_{j k}}{x_{i k}+x_{j k}}\right|$,

where: $x_{i k}, x_{j k}$ are the pair of coordinates of the objects;

3) $A V G_{B E R}=\frac{1}{2} \cdot\left(1-\sqrt{\frac{\Omega}{m+\Omega}}\right)$;

4) if distance( sender, receiver $) \leq d_{0}$ then $m=1.5 ; \Omega=E(r)^{2}=2 \cdot \sigma^{2} ;$ $A V G_{B E R\left(\leq d_{0, m}\right)}=\frac{1}{2} \cdot\left(1-\sqrt{\frac{\Omega}{m+\Omega}}\right)$, else

5) if distance (sender, receiver) $>d_{0}$ and distance (sender, receiver $) \leq d_{1}$ then

$m=0.75 ;$
$A V G_{B E R\left(d_{0} \leq d_{1, m}\right)}=\frac{1}{2} \cdot\left(1-\sqrt{\frac{\Omega}{m+\Omega}}\right)$,

else

6) if distance (sender, receiver) $>d_{1}$ then $m=0.5$;

$A V G_{B E R\left(>d_{1, m}\right)}=\frac{1}{2} \cdot\left(1-\sqrt{\frac{\Omega}{m+\Omega}}\right)$,

7) Endif;

8) $R B E_{n_{i} t}=$ initial energy - consumed energy, where $R B E_{n_{i} t}$ is a RBE of node $n_{i}$ at a time $t$;
9) $w_{i}\left(R B E_{n_{i} t}\right)=\frac{1}{R B E_{n_{i} t}}$,

weight of the RBE is inversely proportional to the $\mathrm{RBE}$;

10) if $A V G_{B E R(d, m)} \geq \alpha$ and $w_{i}\left(R B E_{n_{i} t}\right) \geq \beta$, then

11) select the optimal forwarding node, otherwise discard the node;

12) $W_{i}($ optimal path $j)=\sum_{j=0}^{n} W_{i}\left(R B E_{n_{i} t}\right)$;

13) Best optimal path $j_{j}=\min \left(W_{i}(\right.$ optimal path $\left.j)\right)$;

14) if Best ${ }_{\text {optimal path }}>1$ then

15) thus, the shortest path is selected among the best optimal path.

\section{Simulation results}

In ITS safety application, there is not much delay in communicating between the vehicles in the network. In CSMA/CA, the node first listens to the channel through the RTS/CTS packets into the channel. If the channel is free, node directly transmits the packet, otherwise it waits for random back off time. If two or more nodes sense the channel free, this causes the hidden terminal problem that increases the collision at the denser territory (Nguyen et al. 2013). Based on the collision, the random back off value is selected which causes unbounded delay, reduces the throughput, consumes additional energy and it finally reduces the throughput of network (Bilstrup et al. 2010). The G-TDMA schedules the time slot for vehicle in a centralized manner by CH or RSU (Almalag et al. 2012). It reduces the hidden terminal problem and collision in the two-way traffic, but it increases the overhead of cluster formation, $\mathrm{CH}$ selection and $\mathrm{CH}$ maintenance in the highly dynamic nature of the network. However, it does not overcome the merges collision issue in two-way traffic (Omar et al. 2013). The SSD-TDMA identifies the license free channel and assign available spectrum to the spectrum 
scarcity area in order to overcome the spectrum deficiency in an urban scenario (Sivaganesan, Karthikeyini 2015). It assigns the slot in a distributed way to avoid collision and to avoid the wastage of bandwidth in two-way unbalanced traffic scenario (Cabric et al. 2006). The drawback of the SSD-TDMA method does not consider the RR in an unpredictable traffic. CLAO-TCP measures the received signal strength by using deterministic methods to predict the reliable node. It assigns the time slot in distributed ways based on message generation time within the communication range by using directional antennas (Shakkottai et al. 2003). This is to avoid the path loss and collision in early stage and to avoid the TCP unnecessary movement to the slow-start. The drawback of the CLAO-TCP method does not consider the signal propagation in unpredictable scenario of environmental condition. The proposed ARS analysis the ABERN-m of node to improve the reliable communication in an unpredictable environment condition. It predicts the residual battery energy of the node to extend the network lifetime and thereby avoid the overuse of the node. It also uses the accurate distance measure method to find out the distance between the vehicles. The NS2 simulation shows the execution of the proposed ARS evaluated by different performance metrics such as energy consumption, RO, PDR, PLR and EED listed in Table 2. The performance of the proposed system ARS is better than the existing approaches of CSMA/CA, G-TDMA, SSD-TDMA, and CLAO-TCP by Network Simulator NS2 version 2.34. The simulation parameters are listed in Table 3.

\subsection{EU}

Performance metric EU measures the energy consumed when the packet is transferred from source to destination in the network. The $x$-axis in the graph represents the speed of vehicle $[\mathrm{m} / \mathrm{s}]$. The $y$-axis in the graph represents the EU [J]. The EU of CSMA/CA is $45 \mathrm{~J}$ at $100 \mathrm{~m} / \mathrm{s}$, G-TDMA is $40 \mathrm{~J}$ at $100 \mathrm{~m} / \mathrm{s}, \mathrm{SSD}-\mathrm{TDMA}$ is $37 \mathrm{~J}$ at $100 \mathrm{~m} / \mathrm{s}$, and CLAO-TCP is $29 \mathrm{~J}$ at $100 \mathrm{~m} / \mathrm{s}$. The proposed approach ARS is $25 \mathrm{~J}$ at $100 \mathrm{~m} / \mathrm{s}$. The graph proves that the EU of the proposed approach ARS is lesser than the EU of the existing methods and its performance is better than the existing approaches as seen in Figure 8.
Table 3. Simulation parameters

\begin{tabular}{|l|c|}
\hline \multicolumn{1}{|c|}{ Parameters } & Value \\
\hline Simulation time & $60 \mathrm{~s}$ \\
\hline Transmission range & $250 \mathrm{~m}$ \\
\hline Number of channels & 7 \\
\hline Number of vehicles & 100 \\
\hline RSU & 6 \\
\hline Base station & 1 \\
\hline ABER $\alpha$ & $0 \leq \alpha \leq 0.3$ \\
\hline Residual battery weight $\beta$ & $12 \mathrm{~V}$ \\
\hline Channel data rate & $3 \mathrm{mb} / \mathrm{s}$ \\
\hline Packet size & $512 \mathrm{bytes}$ \\
\hline
\end{tabular}

\subsection{RO}

The $\mathrm{RO}$ is measured by the number of control packets required from source to destination in the network. The $x$-axis in the graph represents the speed of vehicle $[\mathrm{m} / \mathrm{s}]$ and the $y$-axis in the graph represents the control overhead [bytes]. The RO of CSMA/CA is 8.3 bytes at $100 \mathrm{~m} / \mathrm{s}$, G-TDMA is 7.2 bytes at $100 \mathrm{~m} / \mathrm{s}$, SSD-TDMA is 6.7 bytes at $100 \mathrm{~m} / \mathrm{s}$, and CLAO-TCP is 3.9 bytes at $100 \mathrm{~m} / \mathrm{s}$. The proposed approach ARS is 3.2 bytes at 100 /s. The graph proves that the $\mathrm{RO}$ of the proposed approach ARS is lesser than the RO of the existing methods and its performance is better than the existing methods as seen in Figure 9.

\subsection{PDR}

The PDR is measured by the ratio of total number of receiving packets to the total number of sending packets from source to destination in the network. The $x$-axis in the graph represents the speed of vehicles in meters per second and the $y$-axis in the graph represents the packet received in bytes. The PDR of CSMA/CA is $76 \%$ at $100 \mathrm{~m} / \mathrm{s}, \mathrm{G}-\mathrm{TD}-$ MA is $79 \%$ at $100 \mathrm{~m} / \mathrm{s}$, SSD-TDMA is $80 \%$ at $100 \mathrm{~m} / \mathrm{s}$ and CLAO-TCP is $83 \%$ at $100 \mathrm{~m} / \mathrm{s}$. The PDR of the proposed approach ARS is $85 \%$ at $100 \mathrm{~m} / \mathrm{s}$. The graph proves that the PDR of the proposed approach ARS is more than the PDR of the existing methods and its performance is better than the existing methods as seen in Figure 10.

Table 2. Performance metrics

\begin{tabular}{|c|l|l|}
\hline No & \multicolumn{1}{|c|}{ Performance metrics } & \multicolumn{1}{c|}{ Formula } \\
\hline 1. & EU & $=\frac{\text { consumed energy }}{\text { speed of vehicle }}$ \\
\hline 2. & Delay & $=$ packet departure time - packet arrival time \\
\hline 3. & Packet deliver ratio & $=\frac{\text { number of packets received }}{\text { number of packet sent }} \cdot 100$ \\
\hline 4. & Control overhead & $=\frac{\text { number of control route request }+ \text { number of route reply }}{\text { total control packet sent }}$ \\
\hline 5. & PLR & $=$ number of packet sent - number of packets received \\
\hline
\end{tabular}




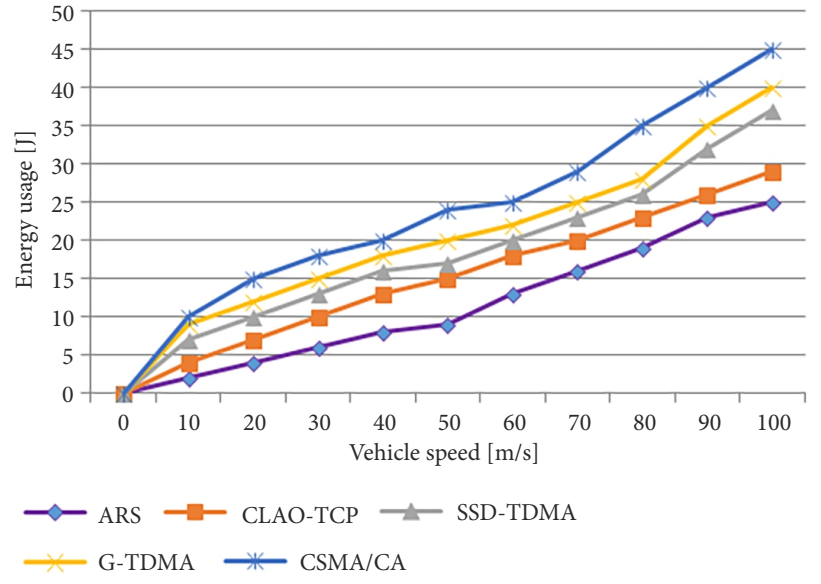

Figure 8. EU

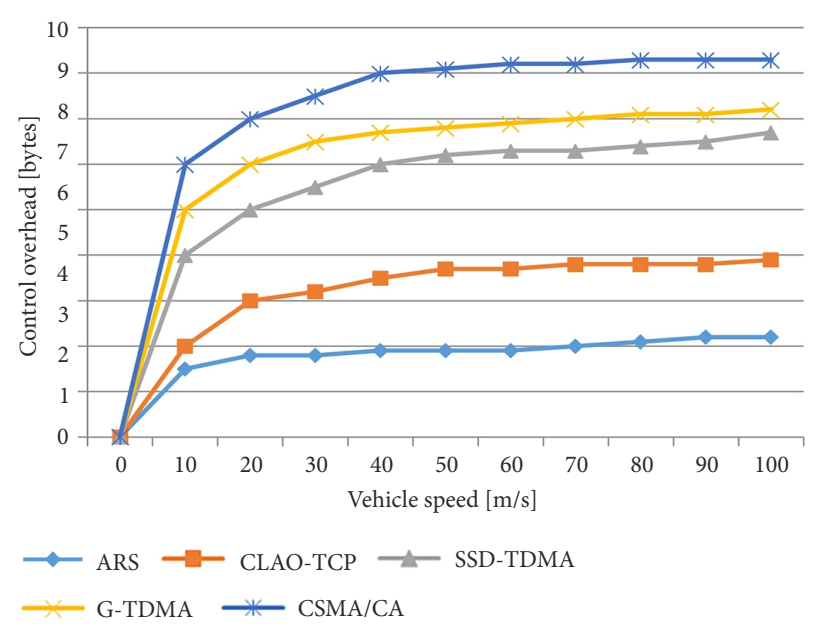

Figure 9. RO

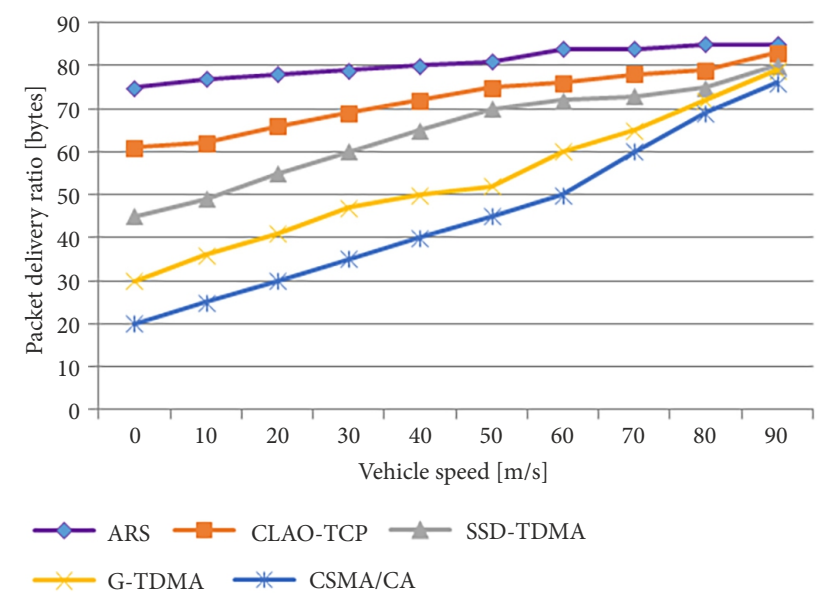

Figure 10. PDR

\subsection{PLR}

The PLR is measured by the difference between the number of packets sent and number of packets received from source to destination in the network. The $x$-axis in the graph represents the speed of vehicle in meters per second

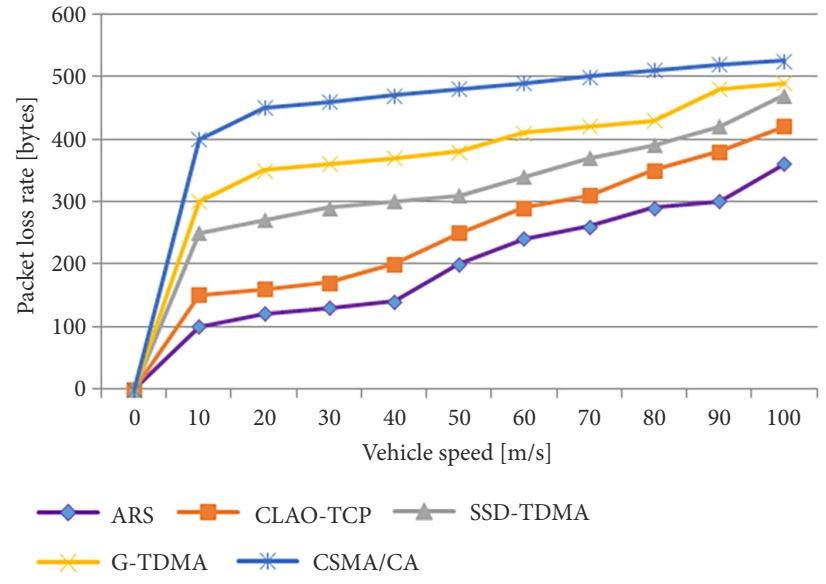

Figure 11. PLR

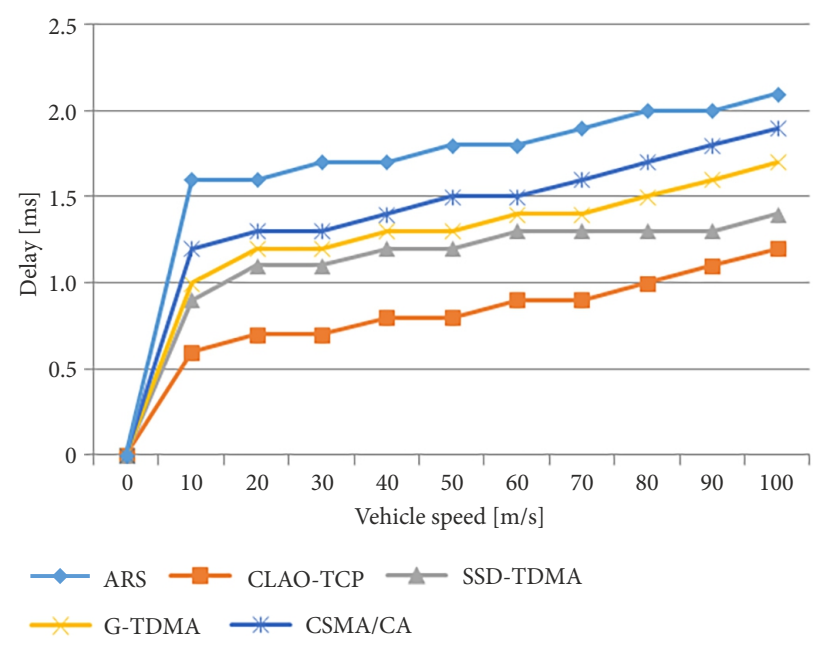

Figure 12. EED

and the $y$-axis in the graph represents the PLR [packets]. The PLR of CSMA/CA is 525 packets at $100 \mathrm{~m} / \mathrm{s}$, G-TDMA is 490 packets at $100 \mathrm{~m} / \mathrm{s}$, SSD-TDMA is 470 packets at $100 \mathrm{~m} / \mathrm{s}$ and CLAO-TCP is 420 packets at $100 \mathrm{~m} / \mathrm{s}$. The PLR of the proposed approach ARS is 360 packets at $100 \mathrm{~m} / \mathrm{s}$. The graph proves that PLR of the proposed approach ARS is lesser than the PLR of the existing methods and its performance is better than the existing methods as seen in Figure 11.

\subsection{EED}

The EED is measured by the time taken for the packets to transfer from source to destination in the network. The $x$-axis in the graph represents the speed of vehicles in meters per second and the $y$-axis in the graph represents the delay [ms]. The EED of CSMA/CA is $2.1 \mathrm{~ms}$ at $100 \mathrm{~m} / \mathrm{s}$, G-TDMA is a $1.9 \mathrm{~ms}$ at $100 \mathrm{~m} / \mathrm{s}$, SSD-TDMA is $1.7 \mathrm{~ms}$ at $100 \mathrm{~m} / \mathrm{s}$ and CLAO-TCP is $1.4 \mathrm{~ms}$ at $100 \mathrm{~m} / \mathrm{s}$. The EED of the proposed approach of ARS is $1.2 \mathrm{~ms}$ at $100 \mathrm{~m} / \mathrm{s}$. The graph proves that the EED of the proposed approach of ARS is less than the existing methods and its performance is better than the existing methods as seen in Figure 12. 


\section{Conclusions}

Vehicles within the network are responsible to send the information from source to the destination.

The received signal strength should be of adequate strength to have a good quality of communication and it should be energy efficient to boost the network lifetime.

The proposed ARS considers the ABERN-m fading for a forwarding node to ensure the quality of communication in mobile nodes. It considers EER to calculate the remaining battery capacity of nodes to enhance the network lifetime, and CDM used to measure the accurate distance between mobile nodes.

The performance metrics of the proposed scheme ARS are analysed by Network Simulator NS2 version 2.34. The proposed scheme ARS proves to be more efficient than the existing schemes.

\section{References}

Al-Sultan, S.; Al-Doori, M. M.; Al-Bayatti, A. H.; Zedan, H. 2014. A comprehensive survey on vehicular ad hoc network, Journal of Network and Computer Applications 37: 380-392. https://doi.org/10.1016/j.jnca.2013.02.036

Almalag, M. S.; Olariu, S.; Weigle, M. C. 2012. TDMA clusterbased MAC for VANETs (TC-MAC), in 2012 IEEE International Symposium on a World of Wireless, Mobile and Multimedia Networks (WoWMoM), 25-28 June 2012, San Francisco, CA, US, 1-6.

https://doi.org/10.1109/WoWMoM.2012.6263796

Beaulieu, N. C.; Cheng, C. 2005. Efficient Nakagami-m fading channel simulation, IEEE Transactions on Vehicular Technology 54(2): 413-424. https://doi.org/10.1109/TVT.2004.841555

Bilstrup, K. S.; Uhlemann, E.; Strom, E. G. 2010. Scalability Issues of the MAC Methods STDMA and CSMA of IEEE 802.11p When Used in VANETs, in 2010 IEEE International Conference on Communications Workshops, 23-27 May 2010, Cape Town, South Africa, 1-5.

https://doi.org/10.1109/ICCW.2010.5503941

Bray, J. R.; Curtis, J. T. 1957. An ordination of the upland forest communities of Southern Wisconsin, Ecological Monographs 27(4): 325-349. https://doi.org/10.2307/1942268

Cabric, D.; Tkachenko, A.; Brodersen, R. W. 2006. Experimental study of spectrum sensing based on energy detection and network cooperation, in TAPAS'06: Proceedings of the First International Workshop on Technology and Policy for Accessing Spectrum, 2-5 August 2006, New York, NY, US, 1-8. https://doi.org/10.1145/1234388.1234400

Craig, J. W. 1991. A new, simple and exact result for calculating the probability of error for two-dimensional signal constellations, MILCOM 91: Military Communications Conference, 4-7 November 1991, McLean, VA, US, 2: 571-575. https://doi.org/10.1109/MILCOM.1991.258319

Das, M.; Sahu, B.; Bhanja, U. 2016. Bit error rate analysis of mobile communication network in Nakagami fading channel: interference considerations, International Journal of Electronics and Communication Engineering 10(8): 1156-1159.

https://doi.org/10.5281/zenodo.1127970

Dhanapal, R.; Visalakshi, P. 2015a. A sector based energy efficient adaptive routing protocol for large scale MANET, Research Journal of Applied Sciences, Engineering and Technology 9(7): 478-484. https://doi.org/10.19026/rjaset.9.1429
Dhanapal, R.; Visalakshi, P. 2015b. Efficient clustering protocol based on ant-bee agent for large scale MANET, International Journal of Applied Engineering Research 10(52): 349-361.

Goldsmith, A. 2005. Wireless Communications. Cambridge University Press. $644 \mathrm{p}$. https://doi.org/10.1017/CBO9780511841224

Gomez, J.; Campbell, A. T.; Naghshineh, M.; Bisdikian, C. 2003. PARO: supporting dynamic power controlled routing in wireless ad hoc networks, Wireless Networks 9(5): 443-460. https://doi.org/10.1023/A:1024636132348

Govindan, K.; Zeng, K.; Mohapatra, P. 2011. Probability density of the received power in mobile networks, IEEE Transactions on Wireless Communications 10(11): 3613-3619. https://doi.org/10.1109/TWC.2011.080611.102250

Hartenstein, H.; Laberteaux, L. P. 2008. A tutorial survey on vehicular ad hoc networks, IEEE Communications Magazine 46(6): 164-171. https://doi.org/10.1109/MCOM.2008.4539481

Hrovat, A.; Kandus, G.; Javornik, T. 2014. A survey of radio propagation modeling for tunnels, IEEE Communications Surveys \& Tutorials 16(2): 658-669. https://doi.org/10.1109/SURV.2013.091213.00175

IEEE Standard 802.2-1989. ISO/IEEE International Standard Information Processing Systems - Local Area Networks - Part 2: Logic Link Control.

IEEE Standard 802.11-2016. IEEE Standard for Information Technology - Telecommunications and Information Exchange Between Systems Local and Metropolitan Area Networks Specific requirements - Part 11: Wireless LAN Medium Access Control (MAC) and Physical Layer (PHY) Specifications.

IEEE Standard 802.11a-1999. IEEE Standard for Telecommunications and Information Exchange Between Systems - LAN/ MAN Specific Requirements - Part 11: Wireless Medium Access Control (MAC) and Physical Layer (PHY) Specifications: High Speed Physical Layer in the $5 \mathrm{GHz}$ Band.

IEEE Standard 802.11b-1999. IEEE Standard for Information Technology - Telecommunications and Information Exchange Between Systems - Local and Metropolitan Networks - Specific Requirements - Part 11: Wireless LAN Medium Access Control (MAC) and Physical Layer (PHY) Specifications: Higher Speed Physical Layer (PHY) Extension in the $2.4 \mathrm{GHz}$ Band.

IEEE Standard IEEE 802.11g-2003. IEEE Standard for Information technology - Local and Metropolitan Area Networks Specific Requirements - Part 11: Wireless LAN Medium Access Control (MAC) and Physical Layer (PHY) Specifications: Further Higher Data Rate Extension in the $2.4 \mathrm{GHz}$ Band.

IEEE Standard 802.11p-2010. IEEE Standard for Information Technology - Local and metropolitan area networks - Specific Requirements - Part 11: Wireless LAN Medium Access Control (MAC) and Physical Layer (PHY) Specifications Amendment 6: Wireless Access in Vehicular Environments.

IEEE Standard 1609.2b-2019. IEEE Standard for Wireless Access in Vehicular Environments - Security Services for Applications and Management Messages - Amendment 2 - PDU Functional Types and Encryption Key Management.

IEEE Standard 1609.3-2016. IEEE Standard for Wireless Access in Vehicular Environments (WAVE) - Networking Services.

IEEE Standard 1609.4-2016. IEEE Standard for Wireless Access in Vehicular Environments (WAVE) - Multi-Channel Operation.

Jain, A. K.; Dubes, R. C. 1988. Algorithms for Clustering Data. Pearson College Div. 320 p.

Lance, G. N.; Williams, W. T. 1966. Computer programs for hierarchical polythetic classification ("similarity analyses"), The Computer Journal 9(1): 60-64.

https://doi.org/10.1093/comjnl/9.1.60 
Lever, K. V. 1998. New derivation of Craig's formula for the Gaussian probability function, Electronics Letters 34(19): 1821-1822. https://doi.org/10.1049/el:19981309

Martinez, F. J.; Toh, C.-K.; Cano, J.-C.; Calafate, C. T.; Manzoni, P. 2009. Realistic radio propagation models (RPMs) for VANET simulations, in 2009 IEEE Wireless Communications and Networking Conference, 5-8 April 2009, Budapest, Hungary, 1-6. https://doi.org/10.1109/WCNC.2009.4917932

Nguyen, T. V.; Baccelli, F.; Zhu, K.; Subramanian, S.; Wu, X. 2013. A performance analysis of CSMA based broadcast protocol in VANETs, in 2013 Proceedings IEEE INFOCOM, 14-19 April 2013, Turin, Italy, 2805-2813. https://doi.org/10.1109/INFCOM.2013.6567090

Omar, H. A.; Zhuang, W.; Li, L. 2013. VeMAC: a TDMA-based MAC protocol for reliable broadcast in VANETs, IEEE Transactions on Mobile Computing 12(9): 1724-1736. https://doi. org/10.1109/TMC.2012.142

Rappaport, T. S. 2002. Wireless Communications: Principles and Practice. Prentice Hall. 736 p.

SAE Standard J2735_201603. Dedicated Short Range Communications (DSRC) Message Set Dictionary.

Shakkottai, S.; Rappaport, T. S.; Karlsson, P. C. 2003. Cross-layer design for wireless networks, IEEE Communications Magazine 41(10): 74-80. https://doi.org/10.1109/MCOM.2003.1235598

Shirkhorshidi, A. S.; Aghabozorgi, S.; Wah, T. Y. 2015. A comparison study on similarity and dissimilarity measures in clustering continuous data, Plos One 10(12): e0144059. https://doi.org/10.1371/journal.pone.0144059

Simon, M. K.; Alouini, M.-S. 2008. Digital communications over fading channels, IEEE Transactions on Information Theory 54(7): 3369-3370. https://doi.org/10.1109/TIT.2008.924676

Simon, M. K.; Alouini, M.-S. 2002. Digital Communication over Fading Channels: a Unified Approach to Performance Analysis. Wiley-Interscience. $544 \mathrm{p}$.

Sivaganesan, D.; Karthikeyini, S. 2015. Cooperative spectrum sensing scheme for vehicle to vehicle communication, $A R P N$ Journal of Engineering and Applied Sciences 10(13): 56505654.

Sivasakthi, M.; Suresh, S. R. 2013. Research on vehicular ad hoc networks (VANETs), Journal of Applied Sciences and Engineering Research 2(1): 23-27.

Sussman, J. M.; Bronzini, M. S. 2006. A Review of: "Perspectives on intelligent transportation systems", Journal of Intelligent Transportation Systems: Technology, Planning, and Operations 10(2): 101-102. https://doi.org/10.1080/15472450600626281

Tarique, M.; Hasan, T. 2011. Impact of Nakagami-m fading model on multi-hop mobile ad hoc network, International Journal of Computer Applications 26(2): 5-12. https://doi.org/10.5120/3078-4214

Tepedelenlioglu, C.; Gao, P. 2005. Estimators of the Nakagami-m parameter and performance analysis, IEEE Transactions on Wireless Communications 4(2): 519-527. https://doi.org/10.1109/TWC.2004.843017

Tiwari, K.; Jain, A.; Charhate, S. V. 2009. BER analysis of Nakagami-m channels with different modulation techniques and transmit diversity, in 2009 Proceeding of International Conference on Methods and Models in Computer Science (ICM2CS), 14-15 December 2009, Delhi, India, 1-3. https://doi.org/10.1109/ICM2CS.2009.5397947

Toh, C.-K. 2001. Maximum battery life routing to support ubiquitous mobile computing in wireless ad hoc networks, IEEE Communications Magazine 39(6): 138-147.

https://doi.org/10.1109/35.925682
$\mathrm{Xu}$, R.; Wunsch, D. 2005. Survey of clustering algorithms, IEEE Transactions on Neural Networks 16(3): 645-678. https://doi.org/10.1109/TNN.2005.845141

Yacoub, M. D.; Fraidenraich, G.; Santos Filho, J. C. S. 2005. Nakagami-m phase-envelope joint distribution, Electronics Letters 41(5): 259-261. https://doi.org/10.1049/el:20057014 